OPEN ACCESS

Edited by: Jeff Sakamoto,

University of Michigan, USA

Reviewed by:

Fatih A. Cetinel,

BASF SE, Germany Juchen Guo,

University of California Riverside, USA

*Correspondence:

Conrad R. Stoldt stoldt@colorado.edu

Specialty section:

This article was submitted

to Energy Storage,

a section of the journal

Frontiers in Energy Research

Received: 25 January 2016

Accepted: 21 March 2016

Published: 31 March 2016

Citation:

Lisenker I and Stoldt CR (2016) Improving NASICON Sinterability through Crystallization under High-Frequency Electrical Fields.

Front. Energy Res. 4:13.

doi: 10.3389/fenrg.2016.00013

\section{Improving NASICON Sinterability through Crystallization under High-Frequency Electrical Fields}

\author{
Ilya Lisenker and Conrad R. Stoldt* \\ Department of Mechanical Engineering, University of Colorado Boulder, Boulder, CO, USA
}

The effect of high-frequency (HF) electric fields on the crystallization and sintering rates of a lithium aluminum germanium phosphate (LAGP) ion conducting ceramic was investigated. LAGP with the nominal composition $\mathrm{Li}_{1.5} \mathrm{Al}_{0.5} \mathrm{Ge}_{1.5}\left(\mathrm{PO}_{4}\right)_{3}$ was crystallized and sintered, both conventionally and under effect of electrical field. Electrical field application, of $300 \mathrm{~V} / \mathrm{cm}$ at $1 \mathrm{MHz}$, produced up to a $40 \%$ improvement in sintering rate of LAGP that was crystallized and sintered under the HF field. Heat sink effect of the electrodes appears to arrest thermal runaway and subsequent flash behavior. Sintered pellets were characterized using $\mathrm{X}$-ray diffraction, scanning electron microscope, TEM, and electrochemical impedance spectroscopy to compare conventionally and field-sintered processes. The as-sintered structure appears largely unaffected by the field as the sintering curves tend to converge beyond initial stages of sintering. Differences in densities and microstructure after $1 \mathrm{~h}$ of sintering were minor with measured sintering strains of 31 vs. $26 \%$ with and without field, respectively. Ionic conductivity of the sintered pellets was evaluated, and no deterioration due to the use of HF field was noted, though capacitance of grain boundaries due to secondary phases was significantly increased.

Keywords: NASICON, field-assisted sintering, high frequency, sintering, grain boundary capacitance

\section{INTRODUCTION}

Sodium superionic conducting (NASICON) ceramics are a family of sodium- or lithium-based solids that have been investigated as potential solid-state electrolytes since the 1970s. They offer relatively high room temperature conductivity, improved safety due to their intrinsic stability, and the possibility of assembling mechanically robust monolithic battery cells (Birke et al., 1997; Nagata and Nanno, 2007). Furthermore, as a high modulus and hardness ceramic solid electrolyte, these materials are proposed to inhibit dendrite formation during cycling, thus preventing the possibility of a short circuit, thermal runaway, and ultimately battery cell failure. The leading candidates among this family of ceramics are aluminum substituted lithium titanium and lithium germanium phosphates. Trivalent ion substitution within the NASICON structure requires insertion of additional lithium to maintain charge neutrality. The additional lithium ions, which force a rearrangement of the lithium sublattice, have the effect of dramatically increasing lithium mobility and therefore conductivity. In particular, the $\mathrm{Li}_{1+x} \mathrm{Al}_{x} \mathrm{Ge}_{2-x}\left(\mathrm{PO}_{4}\right)_{3}$ [lithium aluminum germanium phosphate (LAGP)], $x=0.45$ appears to provide the highest conductivity for $\mathrm{Li}^{+}$ions (Li et al., 1988; Francisco et al., 2015), with higher $x$ values resulting in secondary phase formation (Cretin and Fabry, 1999). At a minimum, a dense structure with no interconnected porosity is required to inhibit dendrite propagation in lithium metal battery cells; however, the sintering of LAGP to a consistently high density remains a 
challenge with densities of $70 \%$ to just over $90 \%$ of theoretically reported despite sometimes lengthy sintering cycles (Cretin and Fabry, 1999; Yang et al., 2015).

A number of sintering approaches can be utilized to increase densification rates. Raising sintering temperatures is the simplest approach, but for LAGP above $800-825^{\circ} \mathrm{C}$, phase separation occurs with formation of insulating phases (Thockchom and Kumar, 2010; Mariappan et al., 2011). The use of high heating rates, for example, by spark plasma sintering (SPS), to increase sintering rates has been well described. In addition to the applied mechanical pressure, the material is in intimate contact with a resistively heated graphite die resulting in heating rates in the hundreds of degree Celsius per minute. In materials for which the bulk diffusion activation energy, responsible for densification, is higher than the activation energy for surface diffusion, responsible for grain coarsening, rapid densification occurs (Stanciu et al., 2001). Resistively heating the sample itself, a process limited to electronically conductive materials is even more effective than traditional SPS but requires an SPS furnace with additional complexity of the die structure (Zapata-Solvas et al., 2015). SPS sintering of LAGP has been reported, but the achieved density was only $87 \%$ of theoretical, and the $20^{\circ} \mathrm{C}$ conductivity was measured to be $3.3 \times 10^{-5} \mathrm{~S} \mathrm{~cm}^{-1}$ (Kubanska et al., 2014), a much lower value than what is commonly reported for such a density (Mariappan et al., 2011). It is notable that an additional, though low intensity, peak appears in the X-ray diffraction (XRD) pattern of the sample after SPS, corresponding to the most intense peak of $\mathrm{AlPO}_{4}$, an insulating secondary phase, though a causal connection was not asserted.

Microwave sintering is another effective approach for rapid sintering of ceramic materials. The alternating electrical field component in microwave processing is used to polarize the ceramic, an inherently lossy process, thereby transferring energy to the crystal lattice with every reversal while rapidly heating the material (Sudiana et al., 2013). Notably, the higher frequencies above about $28 \mathrm{GHz}$ that are needed to prevent formation of standing waves in the chamber and resultant hot spots in the material (Sudiana et al., 2013) may prevent its use in a co-sintering application of a battery device, since the bulk of the device would be effectively shielded by any electronically conductive materials (Bokhan et al., 1995).

Flash sintering methods use DC or lower frequency (up to $1000 \mathrm{~Hz}$ ) AC electrical current passing directly through the sample to achieve remarkably rapid densification (Cologna et al., 2010). However, the charge carrier drift speed at the currents involved would inevitably cause NASICON phase breakdown due to local changes in stoichiometry resulting from lithium extraction near the anode and lithium accumulation near the cathode, similar phenomena to what has been shown in work with zirconia (Downs, 2013) and thoria (Goldwater, 1961). In addition, flash sintering, with an ohmic conducting path from the electrodes to the component and a dielectric breakdown-like thermal initiation mechanism (Todd et al., 2015; Zhang et al., 2015), presents a formidable engineering challenge in assuring an even current distribution across essentially $2 \mathrm{D}$ objects, such as battery components.
Finally, we note that a number of investigations have shown that the manner of material synthesis, and especially crystallization parameters, can substantially influence the conductivity of the final product (Thockchom and Kumar, 2010; Yang et al., 2015). Subjecting amorphous materials to alternating electrical fields, microwave irradiation in particular, has been used to promote uniform crystallization (Mahmoud, 2007).

To the authors' knowledge, frequencies between $1 \mathrm{kHz}$ and $2.4 \mathrm{GHz}$ represent an unexplored frequency range for fieldassisted sintering. In this work, we evaluate the use of highfrequency (HF) electrical fields during synthesis, crystallization, and sintering as a means of enhancing sintering rate beyond that found in conventional thermal sintering processes for NASICON type ceramics. The choice of frequencies in the megahertz range represents a compromise between the shallow penetration of microwaves and electrolysis-inducing direct current. We further demonstrate the ability of a HF field to capacitively couple to the sample, eliminating both hot spots, and the need for platinum paints used in flash sintering to achieve a reliable electrical contact to the ceramic specimen.

\section{EXPERIMENTAL PROCEDURE}

\section{Experimental Apparatus}

A custom built set-up, as shown in Figure 1, was designed and fabricated to allow for the application of a variable frequency and variable voltage AC electrical field across the pellet during both crystallization and sintering. The set-up was contained inside a vertical tube furnace (Mellen Co., Concord, NH, USA). The furnace temperature was controlled using a k-type thermocouple embedded within one of the electrodes, approximately $1 \mathrm{~mm}$ below the sample, in an attempt to mitigate temperature gradients and sample self-heating. Any sample self-heating was thereby

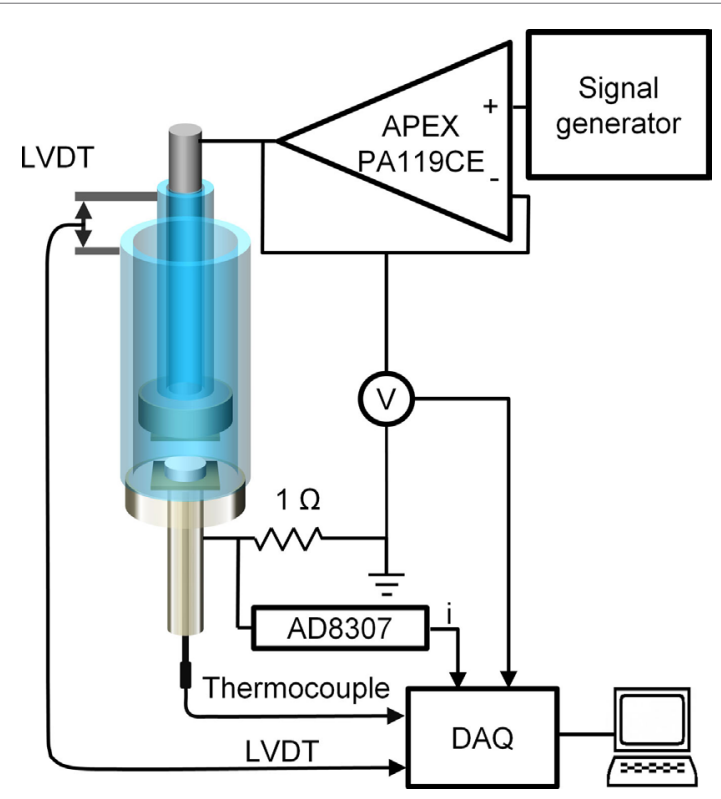

FIGURE 1 | Sintering and data acquisition set-up. 
compensated by the furnace controller ensuring a stable sample temperature to within $2^{\circ} \mathrm{C}$ of the target.

Inconel 625 electrodes were separated from the sample pellet by $0.2-\mathrm{mm}$ thick wafers made of Hexoloy SA silicon carbide (Saint-Gobain Ceramics, France) in order to prevent chromium oxide, deriving from Inconel surface oxidation, from contaminating the pellets. A custom built, all-quartz rod-in-tube dilatometer, based on a GTX-1000 LVDT and S7AC transducer (RDP Group, England), was used to monitor shrinkage in real time. The weight of the dilatometer components exerted an effective pressure of $0.2 \mathrm{MPa}$ on the pellet during crystallization and sintering, thus ensuring intimate contact between electrodes and the pellet. The pressure exerted by the dilatometer was sufficiently low to prevent any sinter-forging of the sample, but was sufficient to prevent radial shrinkage of the pellets. An up to $30-\mathrm{V}$ peak-to-peak, $1-\mathrm{MHz}$ AC field can be applied across the pellet using a custom built amplifier based on APEX PA119CE (Apex Microtechnology, Tucson, AZ, USA) op-amp driven by Agilent 33220A signal generator (Keysight Technologies, Santa Rosa, CA, USA), resulting in a field approximately $300 \mathrm{~V} / \mathrm{cm}$ for a typical 1-mm thick pellet. Note that the capacitor formed by the electrode and the pellet faces acts as a short circuit, inducing a current flow in the sample without any conductive coatings (e.g., platinum) on the pellet faces, as is required for DC and low frequency AC flash sintering experiments. Voltage and current monitoring were provided by a Schottky diode peak detector circuit and AD8307A (Analog Devices, Norwood, MA, USA) logarithmic amplifier to convert high-frequency signal to $0-10 \mathrm{~V}$ analog signal. Data acquisition was performed using U3-HV DAQ (Labjack Corp. Lakewood, $\mathrm{CO}, \mathrm{USA}$ ) at 1-s intervals.

\section{Material Synthesis and Crystallization}

The entire sample preparation process can be followed by referencing the flowchart in Figure 2. Single phase $\mathrm{Li}_{1.5} \mathrm{Al}_{0.5} \mathrm{Ge}_{1.5}\left(\mathrm{PO}_{4}\right)_{3}$

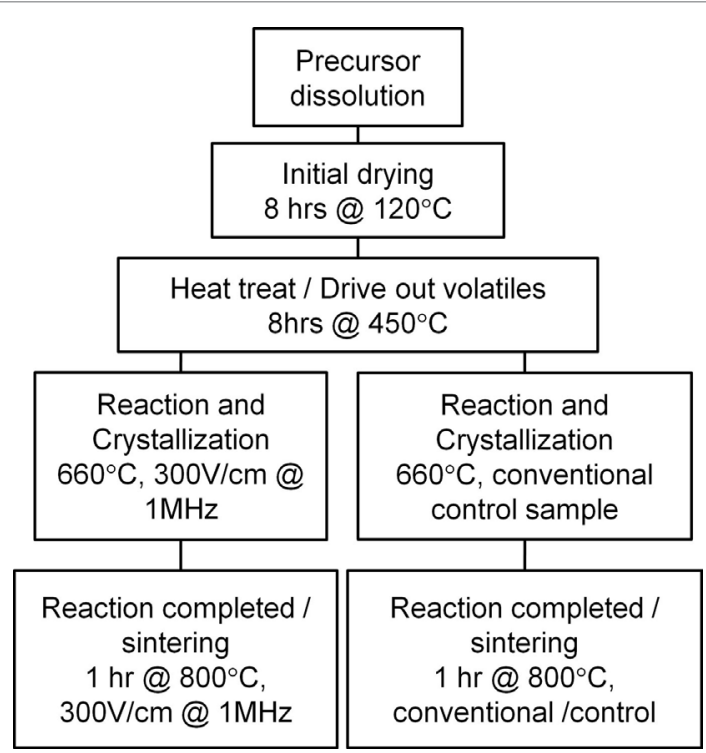

FIGURE 2 | Sample processing flowchart. was prepared by coprecipitation from an aqueous mixture of $\mathrm{LiNO}_{3}$ (Alfa Aesar, 99\%), $\mathrm{AlNO}_{3} \cdot 9 \mathrm{H}_{2} \mathrm{O}$ (Alfa Aesar, 98-102\%), $\left(\mathrm{NH}_{4}\right) \mathrm{H}_{2} \mathrm{PO}_{4}$ (Sigma-Aldrich, 99.99\%), and an ethanol solution of $\mathrm{Ge}(\mathrm{EtOH})_{4}$ (Gelest, 99.99\%). The resulting precipitate was thoroughly dried by heating with stirring for $8 \mathrm{~h}$ at $80^{\circ} \mathrm{C}$ followed by an additional $8 \mathrm{~h}$ at $120^{\circ} \mathrm{C}$. The precipitate was then reground in a mortar and heat treated in an alumina crucible in air for $8 \mathrm{~h}$ at $450^{\circ} \mathrm{C}$ to drive out most of the volatiles, including water and ammonia. The powder was then uniaxially pressed at a pressure of $220 \mathrm{MPa}$ into 70-mg pellets with approximate dimensions of $6.35 \mathrm{~mm}$ diameter by $1.3 \mathrm{~mm}$ thickness. No binder was used for the pressing.

Two subsequent material crystallization routes were pursued. In both routes, the pellets were heat treated in the experimental furnace according to the schedule shown in Figure 3. Half of the material was heat treated under an applied high-frequency (HF) electrical field of $30 \mathrm{~V}$ peak-to-peak amplitude and $1.0 \mathrm{MHz}$ frequency. Control samples were heat treated under identical conditions without HF field. The evolution of pellet thickness during the heat treatment is also illustrated in Figure 3 (note that here positive displacement corresponds to reduction in thickness). The measured volume change coincides with LAGP crystallization peak reported previously by differential scanning calorimetry (Kubanska et al., 2014; Yang et al., 2015). The crystallization process resulted in highly porous bodies of agglomerated crystalline powder that were subsequently reground by hand in a mortar.

\section{Sample Sintering}

After crystallization, the respective LAGP powders were again uniaxially pressed (without binder) at a pressure of $220 \mathrm{MPa}$ into 70-mg pellets with approximate dimensions of $6.35 \mathrm{~mm}$ diameter by $1.0 \mathrm{~mm}$ thickness. All pellets were sintered in a vertical tube furnace in air at $800^{\circ} \mathrm{C}$ for $1 \mathrm{~h}$ with a $10^{\circ} \mathrm{C} / \mathrm{min}$ heating ramp under a $300 \mathrm{~V} / \mathrm{cm}, 1-\mathrm{MHz} \mathrm{HF}$ field. The field was applied at the beginning of the heating ramp and was turned off after $4 \mathrm{~h}$, once the sample had cooled below approximately $100^{\circ} \mathrm{C}$. A total of five

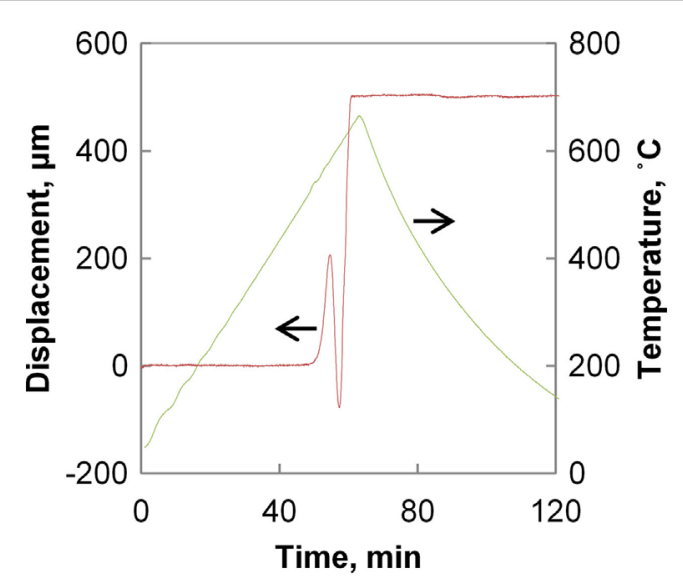

FIGURE 3 | Sintering schedule and displacement (volume change) during the crystallization process. 
replicates from the two different synthesis runs were made. An additional five replicates using the same batch of material were used as controls with no field applied. Post-sintering sample shrinkage was confirmed using digital calipers.

The shorter time interval, the schedule of which is shown in Figure 4, while not allowing for full sintering, serves to accentuate the differences in initial densification rates for the methods used.

\section{Characterization}

Powder at every step of preparation and the sintered samples was characterized by XRD using a Bruker D2 Phaser (Bruker GmbH, Germany) using $\mathrm{Cu}-\mathrm{K}_{\alpha}$ radiation. Raman spectra, not reported here, on pellet faces was acquired using a Jasco NRS-3100 system (Jasco Corp., Tokyo, Japan) at a 532-nm excitation wavelength with a laser power level of approximately $65 \mathrm{~mW}$ and $50 \times$ effective magnification to non-destructively confirm material structure. Sample porosity was determined based on geometric measurement of pellet volume using digital calipers and the LAGP theoretical density as calculated from unit cell parameters.

Sample faces were subsequently sputter coated with approximately $500 \mathrm{~nm}$ of pure gold and electrochemical impedance spectroscopy (EIS) measurements were conducted using a Solartron 1250B potentiostat with a Solartron 1287 electrochemical interface (Ametek, Inc., Farnborough, UK) with a $100-\mathrm{mV}$ excitation amplitude over a frequency range from $0.1 \mathrm{~Hz}$ to $1 \mathrm{MHz}$. EIS measurements were performed in an airtight enclosure under flowing dry nitrogen in a range of temperatures from room temperature down to $-70^{\circ} \mathrm{C}$. Fracture surfaces of two of the pellets were sputter coated with carbon and imaged using JEOL JSM-7401F field emission scanning electron microscope (SEM).

Additionally, two samples were prepared using FIB and imaged using a Talos F200S high-resolution transmission electron microscope (HRTEM) (FEI, Hillsboro, OR, USA) with energy dispersive X-ray spectroscopy (EDS) capability for elemental mapping.

\section{RESULTS AND DISCUSSION}

A comparison of XRD patterns from as-crystallized powders is shown in Figure 5. The powder crystallized under electrical field shows a slightly higher degree of crystallinity with approximately $10 \%$ larger grain size as determined by the Scherrer formula, specifically 69 and $63 \mathrm{~nm}$, respectively. With no apparent differences in the timing and temperature of the onset of the crystallization,

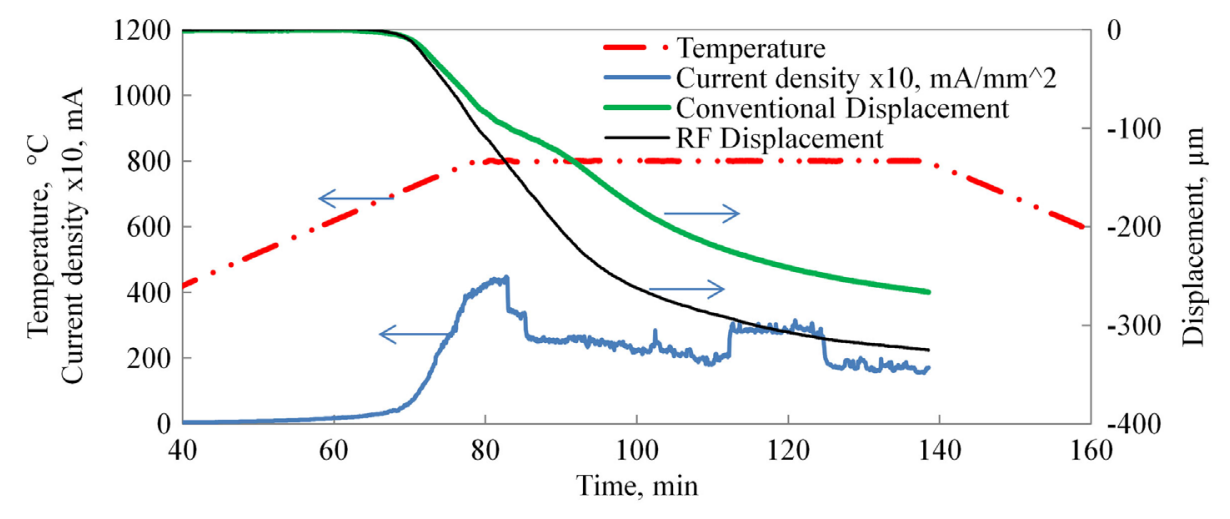

FIGURE 4 | Conventional vs. HF sintering displacement curves. Note that $100-\mu \mathrm{m}$ displacement corresponds to $10 \%$ strain.

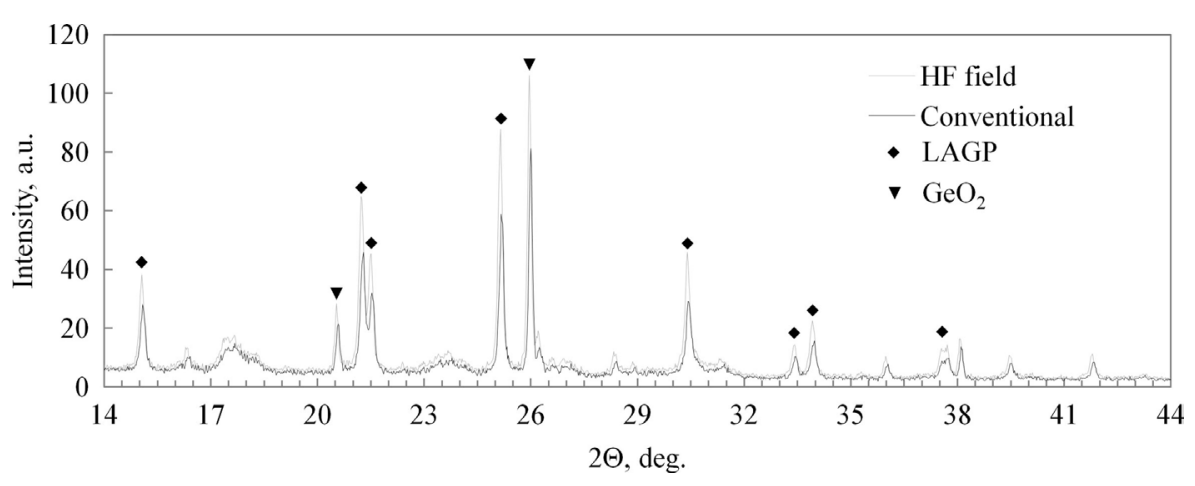

FIGURE 5 | XRD of as-crystallized powders. 
it is reasonable to conclude that the field promotes crystallization through a mild non-thermal effect.

A comparison of the conventional and field-assisted final sintering curves is shown in Figure 4. Both curves are averages of five samples each. Sintering commences at approximately $630^{\circ} \mathrm{C}$ with the sintering rate accelerating and rapidly slowing down as some grain growth occurs, thus reducing sintering pressure. Residual thermal expansion of the dilatometer is subtracted from the data shown. The typical current profile is overlaid on the chart in Figure 4 and illustrates the expected rise in electrical conductivity with temperature. The significant rise in measured current illustrates the effectiveness of using HF fields to achieve capacitive coupling with the material and obviates the need for any added conductive coating. The dramatic fluctuations in current are assignable to secondary phases melting and providing intermittent highly conductive paths until they react with the matrix forming the primary phase. This process can repeat itself many times during HF sintering.

It is notable that any effect on sintering rate is minor. Despite the slightly larger starting grain size, the sintering rate under $\mathrm{HF}$ electrical field is only increased by approximately 30\% before and $40 \%$ after reaching steady state temperature. This brings into question the significance of non-thermally activated matter diffusion mechanisms in the LAGP system. Such effects are widely claimed for a number of electrically assisted sintering processes in other material systems. However, most of the theoretical treatment and experimental work is done on systems where charge carriers simultaneously belong to the crystal lattice. This is not the case for LAGP, and it is possible that polarization of the lattice is a requirement for distinct field effects. The final density of the samples was approximately 80.3 vs. $77.9 \%$, and sintering strain of 30.5 vs. $26.2 \%$ for field processed vs. conventionally sintered samples, respectively. With the applied field being in the hundreds of volt per centimeter, a question of why the sample does not flash sinter has to be addressed. Few experimental geometries allow for direct control of the sample temperature by virtue of aspect ratio and electrical connections utilized. By comparison, our experiments embed a shielded thermocouple within the electrode, in intimate contact with the sample itself. This configuration allows for accurate temperature measurement that is not compromised by the applied field. Another benefit is our ability to control the furnace from the same sensor, in effect using the sample temperature for control. Thus, the sample is actively prevented from proceeding into the thermal runaway condition. This is further aided by the very low aspect ratio of the sample, effectively reducing internal thermal gradients and maximizing the intimate thermal contact with the electrodes, thus allowing the electrodes to serve as effective heat sinks. A similar effect has been reported for the flash sintering of zirconia, where the onset of flash was significantly delayed in low aspect ratio samples (Bichaud et al., 2015). To verify that the absence of flash sintering is a result of the thermal sink effect of the electrode configuration in our apparatus, a single sample was pressed from the same powder in the shape of a dog bone using method taught by Francis et al. (2012) and an attempt was made to sinter the sample using the flash sintering technique. The sample flashed immediately upon application of $300 \mathrm{~V} / \mathrm{cm} \mathrm{DC}$ field at a comparatively low temperature of $400^{\circ} \mathrm{C}$ proving that the material is indeed amenable to flash if allowed to experience thermal runaway. The sample did not densify measurably; however, no further investigation was undertaken since this did not further the current study. Thus, thermal runaway in flash sintering appears to behave similarly to thermal dielectric breakdown (O’Dwyer, 1964).

Typical SEM photographs of fracture surfaces, as shown in Figure 6, demonstrate very similar microstructure, consistent with our density measurements. Grain size can be visually estimated to average at $0.3 \mu \mathrm{m}$ in samples sintered with and without field. It appears that any difference in material that results from crystallization under field is effectively diminished after $1 \mathrm{~h}$ at $800^{\circ} \mathrm{C}$. No abnormal grain growth was observed.

The XRD spectra, as shown in Figure 7, for the two processing methods appear nearly identical with slightly better definition of the weaker peaks, such as [006] and [202] in the HF field sintered material. Besides the primary LAGP phase peaks, only minor features are detected at $26.3^{\circ}$ and $20.6^{\circ}$, identified as [101] and [100] peaks of the $\mathrm{GeO}_{2}$ secondary phase. There is a near consensus in the LAGP community that $\mathrm{AlPO}_{4}$ and some version of lithium phosphate are almost invariably present in amorphous form, and therefore do not produce diffraction peaks in XRD spectra. In order to identify the presence of additional amorphous impurities, HRTEM EDS images of HF field sintered material are shown in Figure 8, and contrasted with HRTEM EDS images of conventionally sintered material shown in Figure 9. These highlight misalignment of nearly perfectly formed tetragonal crystals
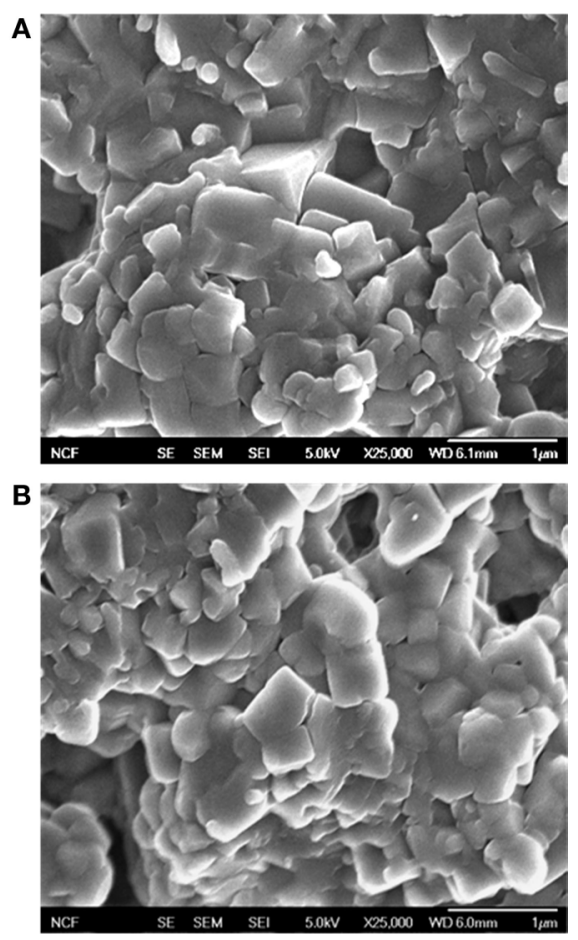

FIGURE 6 | SEM micrographs of selected fracture surfaces. (A) HF sintered and (B) conventionally sintered. 


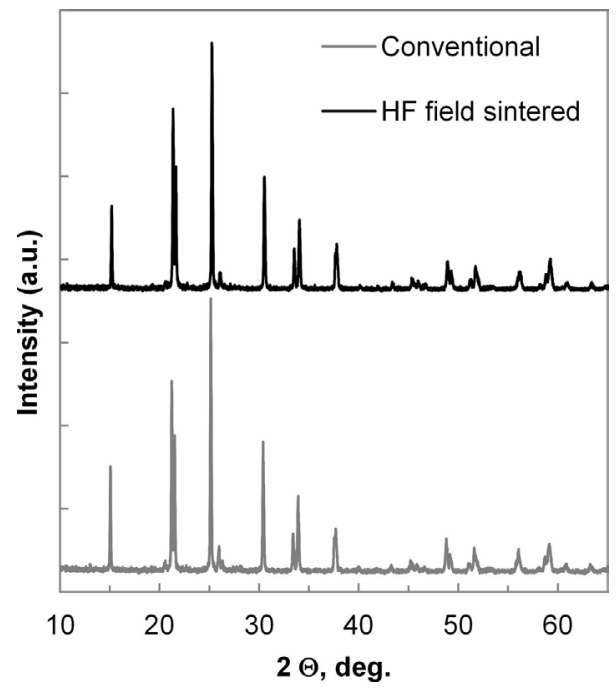

FIGURE 7 | X-ray diffraction patterns for HF (top) and conventionally (bottom) sintered LAGP

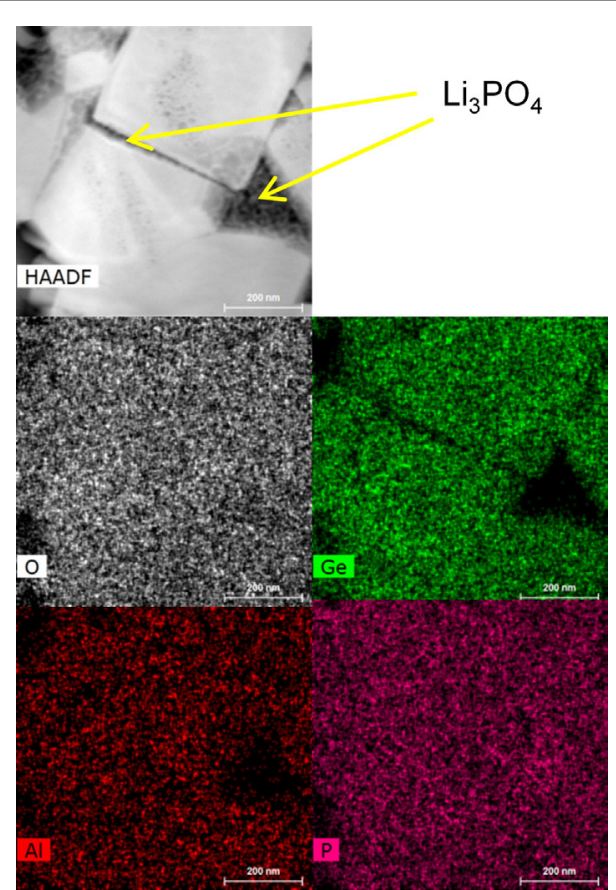

FIGURE 8 | HRTEM, EDS elemental maps, field-assisted sintered sample.

of LAGP with relatively little direct grain-to-grain contact. The amorphous phases between grains are identified by elemental mapping as either lithium phosphate, by absence of $\mathrm{Al}$ or $\mathrm{Ge}$, or as $\mathrm{AlPO}_{4}$. Precise phase identification is challenging as these phases appear extremely vulnerable to electron beam damage, consistent with previous reports (Mariappan et al., 2011). In

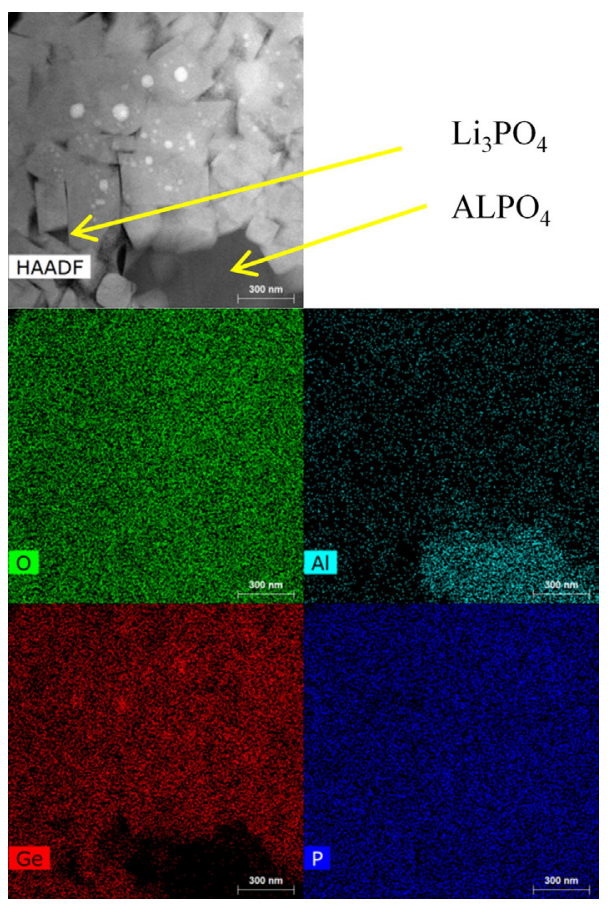

FIGURE 9 | HRTEM, EDS elemental maps, conventionally sintered sample.

general, $\mathrm{AlPO}_{4}$ seems to be more likely to exist as large inclusions or in inter-grain regions with large grain misalignment, whereas lithium phosphate appears to be present in thin layers between mostly aligned grain facets. Due to the much higher $1800^{\circ} \mathrm{C}$ melting point, $\mathrm{AlPO}_{4}$ has a tendency to segregate and form larger inclusions as shown in the image of conventionally sintered LAGP, Figure 9.

Impedance spectroscopy results show essentially identical behavior for samples derived from the two processing methods. A typical Nyquist plot is presented in Figure 10. The expected contributions from bulk and grain boundary conductivity are clearly visible. An RC network model, as shown in the inset in Figure 10, was fit to the data using Zview software (Scribner Associates). Conductivity of the conventional and HF processed material was calculated according to

$$
\sigma=\frac{1}{R} \times \frac{t}{A}
$$

Although the data exhibit some variation among the five samples of each processing type, the distributions overlap between conventionally and field-assisted sintered samples. Typical results for the bulk and grain boundary conductivity are presented in Figure 11 in the form of an Arrhenius plot. The calculated activation energies for the grain and grain boundary conductivity are 0.38 and $0.44 \mathrm{eV}$, respectively, and overall conductivities are on the order of $1.5 \times 10^{-4} \mathrm{~S} / \mathrm{cm}$, consistent with values reported in the literature (Kotobuki et al., 2010). 


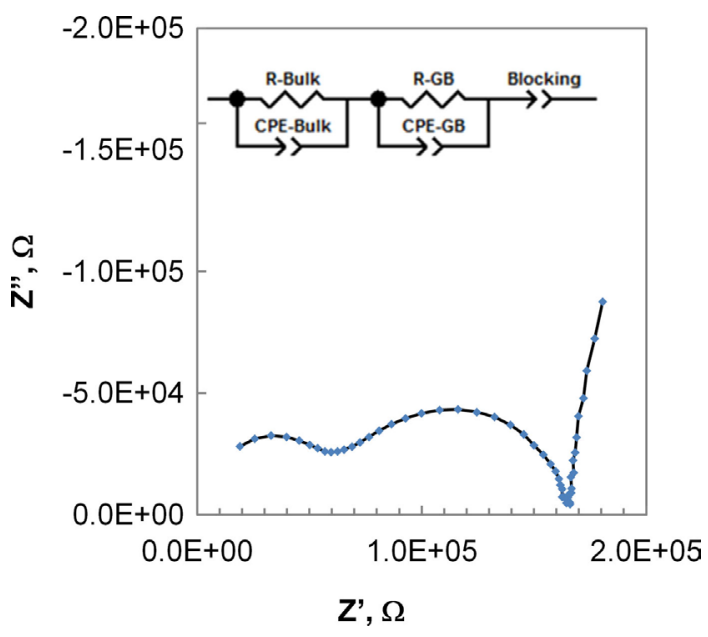

FIGURE 10 | Typical Nyquist plot of the impedance spectrum for a conventionally sintered sample.

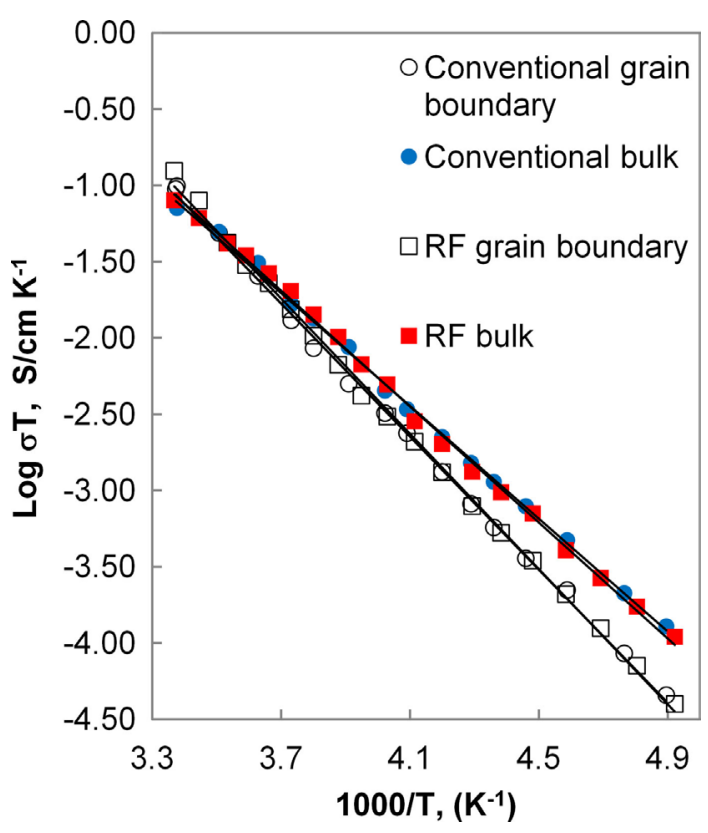

FIGURE 11 | Arrhenius plot of bulk and grain boundary conductivity for conventional and HF sintered samples.

A wholly unexpected effect is the significantly increased grain boundary capacitance of the samples processed under electrical field, as illustrated in Figure 12. It may be tempting to ascribe this effect to a thinner grain boundary, as thinner grain boundaries were convincingly demonstrated in flash sintered zirconia (M'Peko et al., 2013). However, the nearly identical grain boundary conductivity values between fieldassisted and conventional sintering would suggest otherwise. We propose that the cause of the increased grain boundary capacitance is an increased contact area of an insulating

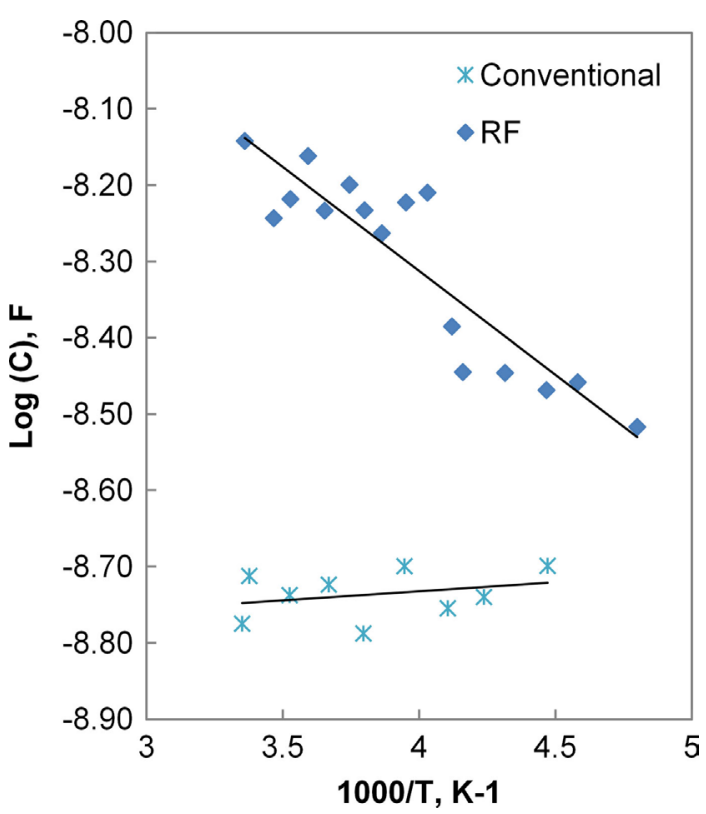

FIGURE 12 | Grain boundary capacitance of typical conventionally and HF sintered samples.

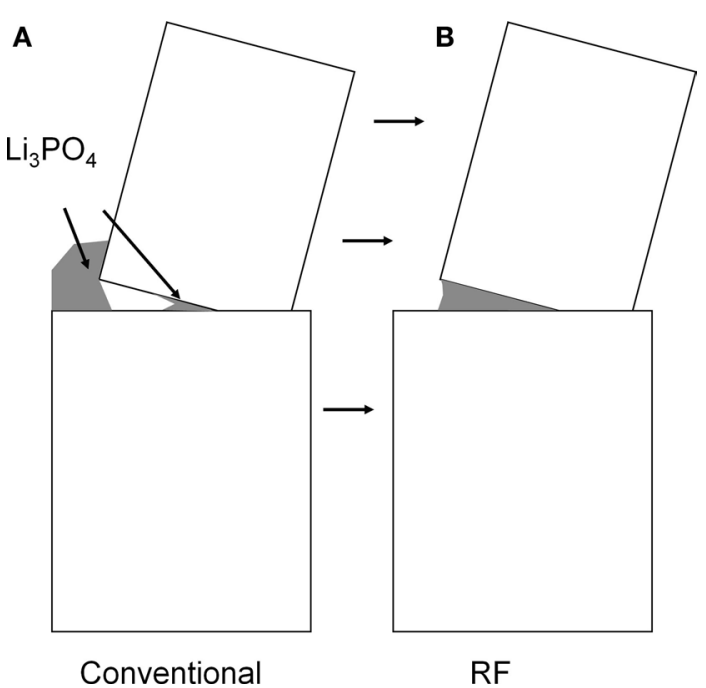

FIGURE 13 | Proposed geometry of grain boundary electrowetting: (A) conventional sintering and (B) field-assisted sintering

intergranular phase adjacent to the actual conducting grain boundary. At least one of the secondary phases, e.g., lithium phosphate, melts at $847^{\circ} \mathrm{C}$ and might form a lower melting eutectic. If melting of these phases occurs at grain boundaries, application of electrical field would lead to electrowetting, effectively pulling these liquid phases into regions of highest field intensity where they subsequently act as a capacitive element in parallel with the grain boundary resistance, as illustrated in Figure 13. 
The HRTEM images from our study and other researchers suggest that LAGP itself is fairly resistant to densification with actual grain contact area being substantially lower than the grain facet area (Mariappan et al., 2011). Thus, the classic brick layer model may be inadequate to explain grain boundary behavior in these materials with abundant secondary phases. In addition, since NASICON crystal conductivity is anisotropic, the widely reported grain conductivity is, at best, the average of a randomly oriented ensemble. The presence of intergranular spaces with a random distribution of insulating, e.g., $\mathrm{Ge}_{2} \mathrm{O}$ and $\mathrm{AlPO}_{4}$, and ionically conductive phases in addition to substantial porosity complicates the ability to rigorously characterize conduction mechanisms. Our future work will focus on separating the contribution of each phase to sinterability and conductivity with an eventual goal of engineering higher performing materials through intelligent grain boundary control.

\section{CONCLUSION}

High-frequency electrical fields by themselves do not appear to have a pronounced effect on sintering rate of LAGP ceramic. However, by allowing the starting material to crystallize under the same field, LAGP does show a moderately increased (+40\%) sintering rate when sintered under field, as compared to powders that were conventionally calcined. Providing efficient heat sinks for Joule heating appears to arrest any tendency toward thermal

\section{REFERENCES}

Bichaud, E., Chaix, J. M., Carry, C., Kleitz, M., and Steil, M. C. (2015). Flash sintering incubation in $\mathrm{Al}_{2} \mathrm{O}_{3} /$ TZP composites. J. Eur. Ceram. Soc. 35, 2587-2592. doi:10.1016/j.jeurceramsoc.2015.02.033

Birke, P., Salam, F., Döring, S., and Weppner, W. (1997). A first approach to a monolithic all solid state inorganic lithium battery. Solid State Ionics118, 149-157. doi:10.1016/S0167-2738(98)00462-7

Bokhan, Yu. I., Komar, V. G., Misyuvyanets, V. Z., Mikhnevich, V. V., and Saraseko, M. N. (1995). High frequency sintering of multilayer ceramic capacitors. J. Eng. Phys. Thermophys. 68, 135-137. doi:10.1007/BF00854378

Cologna, M., Rashkova, B., and Raj, R. (2010). Flash sintering of nanograin zirconia in $<5 \mathrm{sec}$ at 850 degrees C. J. Am. Ceram. Soc. 93, 3556-3559. doi:10.1111/j.1551-2916.2010.04089.x

Cretin, M., and Fabry, P. (1999). Comparative study of lithium ion conductors in the system $\operatorname{LiAlA}\left(\mathrm{PO}_{4}\right)_{3}$ with $\mathrm{A}=\mathrm{Ti}$ or $\mathrm{Ge}$ and $0 \leq \mathrm{x} \leq 0.7$ for use as $\mathrm{Li}+$ sensitive membranes. J. Eur. Ceram. Soc. 19, 2931-2940. doi:10.1016/ S0955-2219(99)00055-2

Downs, J. A. (2013). Mechanisms of Flash Sintering in Cubic Zirconia. Ph.D. dissertation, University of Trento, Trento.

Francis, J. S. C., Cologna, M., and Raj, R. (2012). Particle size effects in flash sintering. J. Eur. Ceram. Soc. 32, 3129-3136. doi:10.1016/j.jeurceramsoc.2012.04.028

Francisco, B. E., Stoldt, C. R., and M'Peko, J.-C. (2015). Energetics of ion transport in NASICON-type electrolytes. J. Phys. Chem. C 119, 16432-16442. doi:10.1021/ acs.jpcc.5b03286

Goldwater, D. L. (1961). The electrolysis of thorium oxide crystals. J. Phys. Chem. Solids 18, 259-260. doi:10.1016/0022-3697(61)90172-X

Kotobuki, M., Hoshina, K., Isshiki, Y., and Kanamura, K. (2011). Preparation of Li1.5Al0.5Ge1.5(PO4)3 solid electrolyte by sol-gel method. Phosphorus Res. Bull. 25, 061-063.

Kubanska, A., Castro, L., Tortet, L., Schäf, O., Dollé, M., and Bouchet, R. (2014). Elaboration of controlled size $\mathrm{Li}_{1.5} \mathrm{Al}_{5} \mathrm{Ge}_{1.5}\left(\mathrm{PO}_{4}\right)_{3}$ crystallites from glass-ceramics. Solid State Ionics 266, 44-50. doi:10.1016/j.ssi.2014.07.013 runaway and flash sintering. Microstructure of the ceramic and its ionic conduction properties also show no negative effects from application of HF electric fields. Finally, we demonstrate a HF field's ability to efficiently couple capacitively into ionically conductive ceramics, preserving stoichiometry and foregoing the need for electrically conductive coatings prior to field-assisted sintering.

\section{AUTHOR CONTRIBUTIONS}

CS provided guidance in experiment design and sample characterization, contributed to and edited the manuscript, and provided final approval for submission. IL designed and built the experimental set-up, manufactured samples for characterization, performed data analysis, assembled the manuscript, and provided final approval for submission.

\section{ACKNOWLEDGMENTS}

Support for instrumentation development was provided under ARPA-E 0869-1584 through a subcontract from Solid Power, Inc. (Louisville, CO, USA). Support for materials characterization was provided under the NSF Sustainable Energy Pathways Program (Project No. DMR-1231048). Additionally, the authors acknowledge the assistance of Dr. David Diercks for the HRTEM analyses and Dr. Brian Francisco for numerous discussions.

Li, S. C., Cai, J. Y., and Lin, Z. X. (1988). Phase relationships and electrical conductivity of LAGP and LACP systems. Solid State Ionics 2, 1265-1270. doi:10.1016/0167-2738(88)90368-2

Mariappan, C. R., Yada, C., Rosciano, F., and Roling, B. (2011). Correlation between micro-structural properties and ionic conductivity of $\mathrm{Li}_{1.5} \mathrm{Al}_{0.5} \mathrm{Ge}_{1.5}\left(\mathrm{PO}_{4}\right)_{3}$ ceramics. J. Power Sources 196, 6456-6464. doi:10.1016/j. jpowsour.2011.03.065

Mahmoud, M. M. (2007). Crystallization of Lithium Disilicate Glass Using Variable Frequency Microwave Processing. Master's thesis, Virginia Polytechnic Institute and State University, Blacksburg, VA.

M'Peko, J.-C., Francis, J. S. C., and Raj, R. (2013). Impedance spectroscopy and dielectric properties of flash versus conventionally sintered yttria-doped zirconia electroceramics viewed at the microstructural level. J. Am. Ceram. Soc. 96, 3760-3767. doi:10.1111/jace.12567

Nagata, N., and Nanno, T. (2007). All solid battery with phosphate compounds made through sintering process. J. Power Sources 174, 832-837. doi:10.1016/j. jpowsour.2007.06.227

O'Dwyer, J. J. (1964). The Theory of Dielectric Breakdown. Oxford: Clarendon Press.

Stanciu, L. A., Kodash, V. Y., and Groza, J. R. (2001). Effects of heating rate on densification and grain growth during field-assisted sintering of $\mathrm{Al}_{2} \mathrm{O}_{3}$ and $\mathrm{MoSi}_{2}$. Metall. Mater. Trans. A 32, 2633-2638. doi:10.1007/s11661-001-0053-6

Sudiana, I. N., Ito, R., Inagaki, S., Kuwayama, K., Sako, K., and Mitsudo, S. (2013). Densification of alumina ceramics sintered by using submillimeter wave gyrotron. J. Infrared, Millim. Terahertz Waves 34, 627-638. doi:10.1007/ s10762-013-0011-6

Thockchom, J. S., and Kumar, B. (2010). The effect of crystallization parameters of the ionic conductivity of a lithium aluminum germanium phosphate glass-ceramic. J. Power Sources 195, 2870-2876. doi:10.1016/j.jpowsour.2009. 11.037

Todd, R. I., Zapata-Solvas, E., Bonilla, R. S., Sneddon, T., and Wilshaw, P. R. (2015). Electrical characteristics of flash sintering: thermal. J. Eur. Ceram. Soc. 35, 1865-1877. doi:10.1016/j.jeurceramsoc.2014.12.022 
Yang, J., Huang, Z., Huang, B., Zhou, J., and Xu, X. (2015). Influence of phosphorus sources on lithium ion conducting performance in the system of $\mathrm{Li}_{2} \mathrm{O}-\mathrm{Al}_{2} \mathrm{O}_{3}$ $\mathrm{GeO}_{2}-\mathrm{P}_{2} \mathrm{O}_{5}$ glass-ceramics. Solid State Ionics 270, 61-65. doi:10.1016/j. ssi.2014.12.013

Zapata-Solvas, E., Gomez-Garcia, D., Dominguez-Rodriguez, A., and Todd, R. I. (2015). Ultra-fast and energy-efficient sintering of ceramics by electric current concentration. Sci. Rep. 5, 1-7. doi:10.1038/srep08513

Zhang, Y., Jung, J., and Luo, J. (2015). Thermal runaway, flash sintering and asymmetrical microstructural development of $\mathrm{ZnO}$ and $\mathrm{ZnO}-\mathrm{Bi}_{2} \mathrm{O}_{3}$ under direct currents. Acta. Mater. 94, 87-100. doi:10.1016/j.actamat.2015.04.018
Conflict of Interest Statement: The corresponding author CS is a founder and member of the Board of Solid Power, Inc., one of the two sponsors of this research work. IL has no conflict of interest to declare.

Copyright (C) 2016 Lisenker and Stoldt. This is an open-access article distributed under the terms of the Creative Commons Attribution License (CC BY). The use, distribution or reproduction in other forums is permitted, provided the original author(s) or licensor are credited and that the original publication in this journal is cited, in accordance with accepted academic practice. No use, distribution or reproduction is permitted which does not comply with these terms. 